\title{
Gamma-rays from the vicinity of accreting neutron stars inside compact high-mass X-ray binaries
}

\begin{abstract}
W. Bednarek
Department of Astrophysics, University of Łódź, 90-236 Łódź, ul. Pomorska 149/153, Poland

e-mail: bednar@fizwe4.phys.uni .lodz.pl

Received 1 October 2008 / Accepted 22 November 2008

\section{ABSTRACT}

The dense wind of a massive star can be captured partially by a neutron star (NS) inside a compact binary system. Depending on the parameters of the NS and the wind, the matter can penetrate the inner NS magnetosphere. At some distance from the NS, a turbulent and magnetized transition region is formed due to the balance between the magnetic pressure and the pressure inserted by accreting matter. This region provides good conditions for acceleration of particles to relativistic energies. The matter at the transition region can farther accrete onto the NS surface (the accretor phase) or be expelled from the NS vicinity (the propeller phase). We consider the consequences of the acceleration of electrons at the transition region, concentrating on the situation in which at least part of the matter falls onto the NS surface. This matter creates a hot spot on the NS surface which emits thermal radiation. Relativistic electrons lose energy to the synchrotron process and the inverse Compton (IC) scattering of this thermal radiation. We calculate the synchrotron spectra (from X-rays to soft $\gamma$-rays) and IC spectra (above a few tens of $\mathrm{MeV}$ ) expected in this scenario. We argue that a population of massive binaries discovered by the INTEGRAL observatory, which contain neutron stars hidden inside dense stellar winds of massive stars, is detectable by the Fermi LAT telescope in the GeV energy range. As an example, we predict the expected $\gamma$-ray flux from the source IGR J19140+0951.
\end{abstract}

Key words. radiation mechanisms: non-thermal - gamma-rays: theory - X-rays: binaries - stars: binaries: close - stars: neutron

\section{Introduction}

Massive binary systems, containing a compact object (neutron star or black hole), have long been suspected as being the sites of particle acceleration up to at least $\mathrm{TeV}$ energies. Three different type of these objects have now been confirmed as VHE $\gamma$-ray sources by Cherenkov telescopes. It is clear that at least in one case the $\gamma$-ray emission is due to the acceleration of particles as a result of the interaction of the energetic pulsar wind with the wind of a massive star. Other $\mathrm{TeV} \gamma$-ray binaries operate either under this same general scenario or the acceleration of particles occurs in the jet launched by the compact object. In the case of these $\mathrm{TeV} \gamma$-ray binaries, the neutron star (pulsar) should rotate sufficiently fast to produce an energetic pulsar wind.

In this paper we consider the case of the neutron star is not so energetic that the matter from the stellar wind can penetrate into the inner pulsar magnetosphere. At certain distances from the NS surface, the magnetic pressure balances the pressure of accreting matter, creating a turbulent transition region. The NS is then enshrouded in a dense cocoon resembling objects called hidden pulsars (see e.g. Tavani 1991; Tavani \& Brookshow 1993) or cauldrons (Begelman \& Rees 1984; Treves et al. 1993). We note that $\gamma$-ray emission was predicted from hidden pulsars (Tavani 1993). We study the case in which the electrons are accelerated inside a turbulent, strongly-magnetized, transition region within the inner pulsar magnetosphere. In principle, electrons can reach even $\sim \mathrm{TeV}$ energies but, as we show below, due to significant synchrotron energy losses, only $\gamma$-rays in the Fermi LAT telescope energy range may be effectively produced.

The INTEGRAL observatory appears to have discovered a class of compact, high-mass X-ray binaries (see e.g.
Chaty et al. 2008; Rodriquez \& Bodaghee 2008) that exhibit a mechanism discussed in this paper. These newly discovered massive binaries are compact with orbital periods between a few to several days. Some contain relatively slowly rotating neutron stars that may allow the matter to penetrate the inner NS magnetosphere. According to the classification scheme of X-ray binaries (e.g. Lipunov 1992). These binaries with slowly rotating neutron stars (NS) belong to the propeller and accretor class. In these objects, the matter of the dense stellar wind accretes onto the strongly magnetized NS, interacting with the rigidly rotating inner magnetosphere. In the case of an accretor, most of the matter reaches the NS surface. In the case of a propeller, most of the matter is expelled from the NS vicinity, due to the centrifugal force.

We propose that this general scenario can be applied to a type of obscured compact objects inside massive binaries that has been observed by the INTEGRAL observatory. We perform detailed calculations of the $\gamma$-rays spectra, including in some cases the inverse Compton (IC) $\mathrm{e}^{ \pm}$pair cascade initiated by relativistic electrons in the radiation field produced on the neutron-star surface. We also account for the synchrotron energy losses of primary electrons and cascade $\mathrm{e}^{ \pm}$pairs, to obtain simultaneous synchrotron X-ray spectra. In this scenario, the acceleration of hadrons and the possible production of high energy $\gamma$-rays, neutrinos, and neutrons will be discussed in another paper.

\section{Description of the model}

We consider a compact binary system containing a rotating neutron star (NS) and a massive, companion O,B type star. It is assumed that mass from the stellar wind is effectively captured by the strong gravitational potential of the NS. These slowly 
rotating neutron stars appear at a certain stage of the evolution of the binary system due to the loss of angular momentum either by the pulsar mechanism or as a result of the torque exhibited by the matter accreted from the stellar wind. Depending on the rotational period and surface magnetic field of NS, the accretion process onto the NS can occur in different phases. According to the classification scheme of Lipunov (1992), the accretion process can occur in the phase of accretor (for relatively slower rotators) or in the phase of propeller. For energetic, strongly magnetized and short-period, neutron stars, the accretion process does not occur at all since the matter cannot penetrate below the light cylinder radius. In the case of NS in the accretor and propeller phases, matter from the stellar wind penetrates below the light cylinder radius of the rotating NS magnetosphere. This matter extracts rotational energy from the NS as a result of the interaction of free-falling matter with the rigidly rotating inner NS magnetosphere. In this paper, we consider the direct collision of matter from the stellar wind with the NS magnetosphere (see the general scenario depicted in Fig. 1). As a result of this interaction, a turbulent, magnetized transition region is formed. In the case of the accretor, most of the matter falls onto the NS surface creating a small hot region on the NS surface. In the case of the propeller, most of the matter is expelled from the vicinity of the NS. It is expected that the matter might be also able to accrete in this stage. This would, however, be non-stationary form since, due to the gradual accumulation of matter close to the transition region, the pressure of the matter can overcome the pressure from the rotating magnetosphere. It is supposed that some amount of the matter can accrete but most of it is expelled from the binary system (possibly forming larger scale jets?) for some specific transition parameters describing the accretor/propeller scenario.

We note that in some massive binaries (e.g. Her X-1), the accretion of matter can also occur by the Roche lobe overflow. The accretion disk around the NS is then formed before the matter from the disk interacts directly with the NS magnetosphere. In this paper, we do not discuss this possibility but instead concentrate on a simpler quasi-spherical accretion onto magnetized NS of the matter from the dense wind of the massive star. The accretion from the disk can be easily taken into account in our scenario by introducing a geometrical factor that describes the part of the sphere in which the accretion occurs. Therefore, the accretion from the disk corresponds to one of our discussed cases with an effectively higher accretion rate occurring only inside a limited region determined by the thickness of the accretion disk.

We first consider the case of the NS in the phase of pure accretor. The accretion rate of the matter onto the neutron star $\left(\dot{M}_{\text {acc }}=10^{16} M_{16} \mathrm{~g} \mathrm{~s}^{-1}\right)$ can be estimated from the observed (in some cases) thermal X-ray emission $\left(L_{\mathrm{X}}=10^{36} L_{36} \mathrm{erg} \mathrm{s}^{-1}\right)$. $\dot{M}_{\text {acc }}$ and $L_{\mathrm{X}}$ can by related to the known radius and mass of the NS (we assume $R_{\mathrm{NS}} \approx 10^{6} \mathrm{~cm}$ and $M_{\mathrm{NS}}=1.4 M_{\odot}$ ),

$\dot{M}_{\text {acc }} \approx 5 \times 10^{15} L_{36} \mathrm{~g} \mathrm{~s}^{-1}$.

This matter arrives onto the neutron star surface along the magnetic-field lines. The distance at which the magnetic field begins to dominate the dynamics of the matter (the Alfven radius) can be estimated by comparing the magnetic-field energy density with the kinetic-energy density of the matter,

$B_{\mathrm{A}}^{2} / 8 \pi=\rho v_{\mathrm{f}}^{2} / 2$,

where $B_{\mathrm{A}}$ is the magnetic field in the inner neutron-star magnetosphere, $\rho=\dot{M}_{\mathrm{acc}} /\left(\pi R_{\mathrm{A}}^{2} v_{\mathrm{f}}\right)$ is the density of accreting matter, $v_{\mathrm{f}}=\left(2 G M_{\mathrm{NS}} / R_{\mathrm{A}}\right)^{1 / 2}$ is the free fall velocity of accreting matter, $R_{\mathrm{A}}$ is the Alfven radius, and $G$ is the gravitational constant.

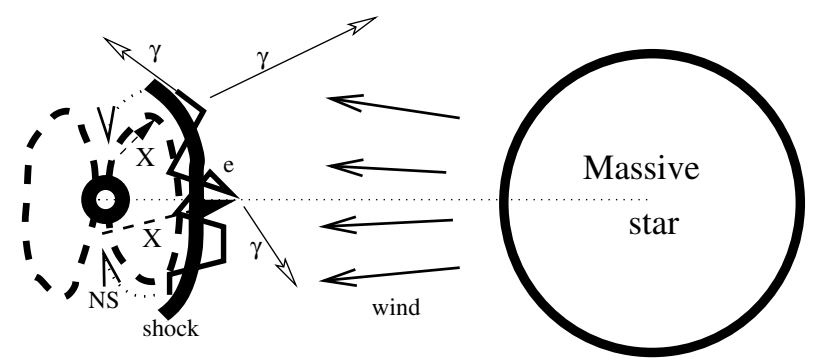

Fig. 1. Schematic representation of the model discussed in this paper. The wind from a massive star penetrates the inner magnetosphere of a neutron star. As a result of the interaction of the wind with the dipole magnetic field of the NS, a strongly magnetized turbulent region is created (thick solid curve) in which the matter gains energy by being accelerated to the velocity of the rotating magnetic-field lines. Electrons (e) are accelerated in this turbulent transition region and cool by means of the synchrotron process and the Comptonization of thermal radiation (marked by X), emitted from the stellar surface around the magneticpole region. As a result, the first generation of IC $\gamma$-rays is produced. Thermal radiation appears due to the gravitational energy release of accreting matter onto the NS surface. If the optical depths for $\gamma$-rays are sufficiently large, they initiate the IC $\mathrm{e}^{ \pm}$pair cascade in this same thermal radiation.

The medium in the transition region is extremely turbulent and strongly magnetized, providing good conditions for the acceleration of particles to high energies. We estimate the location of this region from the surface of the neutron star by applying Eq. (2) and assuming that the magnetic field in the neutron-star magnetosphere is of the dipole type, i.e. $B_{\mathrm{A}}=B_{\mathrm{NS}}\left(R_{\mathrm{NS}} / R_{\mathrm{A}}\right)^{3}$,

$R_{\mathrm{A}}=4 \times 10^{8} B_{12}^{4 / 7} M_{16}^{-2 / 7} \mathrm{~cm}$,

where the magnetic field at the neutron-star surface is $B_{\mathrm{NS}}=$ $10^{12} B_{12}$ (see also Baan \& Treves 1973). Based on the known vale of $R_{\mathrm{A}}$, we can estimate the magnetic-field strength at the transition region,

$B_{\mathrm{A}}=1.6 \times 10^{4} M_{16}^{6 / 7} B_{12}^{-5 / 7} \mathrm{G}$.

We consider that the accretor scenario can occur provided the neutron star fulfills some conditions. First, the radius of the transition region must be inside the light cylinder radius of the neutron star, i.e. $R_{\mathrm{A}}<R_{\mathrm{LC}}=c P / 2 \pi$, where $P=1 P_{1} \mathrm{~s}$ is the rotational period of the neutron star, and $c$ is the velocity of light. The above condition is fulfilled when,

$P_{1}>0.084 B_{12}^{4 / 7} M_{16}^{-2 / 7}$.

Therefore, only relatively slowly rotating neutron stars can be considered.

Second, the rotational velocity of the magnetosphere at $R_{\mathrm{A}}$ must then be lower than the Keplerian velocity of the accreting matter. The rotational velocity,

$v_{\text {rot }}=2 \pi R_{\mathrm{A}} / P \approx 2.5 \times 10^{9} B_{12}^{4 / 7} M_{16}^{-2 / 7} / P_{1} \mathrm{~cm} \mathrm{~s}^{-1}$,

is lower than the Keplerian velocity,

$v_{\mathrm{k}}=\left(G M_{\mathrm{NS}} / R_{\mathrm{A}}\right)^{1 / 2} \approx 6.8 \times 10^{8} B_{12}^{-2 / 7} M_{16}^{1 / 7} \mathrm{~cm} \mathrm{~s}^{-1}$,

for the NS with the periods,

$P_{1}>3.7 B_{12}^{6 / 7} M_{16}^{-3 / 7}$.

This last condition separates the population of NS in the propeller phase (lower periods) from those in the accretor phase 
(larger periods). We note, that the scenario discussed below for acceleration of electrons can also be applied to the propeller stage of accretion onto the NS. However, in the propeller phase, the radiation field created by the matter accreting onto the NS is not uniquely defined. Part of the matter being accreted onto a NS cannot presently be linked to the basic parameters describing the model. We must introduce additional factor describing the amount of the matter that falls onto the NS surface and the total amount of the matter penetrating the inner NS magnetosphere (i.e accreting and being expelled from the vicinity of the NS).

The third condition relates the radius of the transition region, $R_{\mathrm{A}}$, to the capturing radius of the matter from the stellar wind. It is determined by the balance between the kinetic energy of the wind and its gravitational energy around the NS. The capturing radius is described by,

$R_{\mathrm{c}}=2 G M_{\mathrm{NS}} / v_{\mathrm{w}}^{2} \approx 3.7 \times 10^{10} v_{8}^{-2} \mathrm{~cm}$,

where $v_{\mathrm{w}}=10^{8} v_{8} \mathrm{~cm} \mathrm{~s}^{-1}$ is the velocity of surrounding matter measured with respect to the NS. The accretion from the stellar wind occurs when $R_{\mathrm{c}}>R_{\mathrm{A}}$, which happens for,

$B_{12}<2.8 \times 10^{3} v_{8}^{-7 / 2} M_{16}^{1 / 2}$.

For likely parameters of the neutron stars (classical and millisecond pulsars), this limit is not restrictive. We note that $v_{\mathrm{w}}$ corresponds to the velocity of the stellar wind and/or the velocity of the neutron star in its orbit around the massive star. This final velocity can be estimated from $v_{\mathrm{NS}}=2 \pi D / T=$ $5 \times 10^{7} D_{2} / T_{10} \mathrm{~cm} \mathrm{~s}^{-1}$, where $D=100 \cdot D_{2} R_{\odot} \mathrm{cm}$ is the radius of the orbit in units of 100 solar radii $R_{\odot}$, and $T=10 \cdot T_{10}$ days is the period of the binary system in units of 10 days. The velocity is typically lower than the free fall velocity at the $R_{\mathrm{A}}$, and therefore is neglected when deriving Eq. (3).

Neutron stars of periods within the range defined by Eqs. (5) and (8) are in the propeller phase, those of period above that given by Eq. (8) are in the accretor phase, and those of period shorter than given by Eq. (5) are in the ejector phase. As already noted, the accretion rate can be linked directly to the thermal X-ray emission from the NS surface only in the accretor phase. In the propeller phase, the amount of the matter accreting onto the NS surface cannot be uniquely determined from the basic parameters of the model.

\subsection{Acceleration of electrons}

In the conditions expected for the transition region (a strongly magnetized and turbulent medium), particles should be efficiently accelerated. In this paper, we consider only the acceleration of electrons. The energy gain rate of electrons with energy $E$ (and the Lorentz factor $\gamma$ ) is often parametrized by the Larmor radius of electrons and the so-called acceleration parameter,

$\dot{P}_{\mathrm{acc}}=\xi c E / r_{\mathrm{L}} \approx 2.6 \times 10^{4} \xi_{-1} M_{16}^{6 / 7} B_{12}^{-5 / 7} \mathrm{erg} \mathrm{s}^{-1}$,

where $\xi=10^{-1} \xi_{-1}$ is the acceleration parameter, $c$ is the velocity of light, $r_{\mathrm{L}}=E / e B_{\mathrm{A}}$ is the Larmor radius, and $e$ is the electron charge. The acceleration parameter contains all the unknown details of the acceleration process. Since the plasma moves within the transition region at a velocity that is a significant part of the velocity of light, it seems proper to consider the values of $\xi$ in the range $\sim 0.1-0.01$. During the acceleration process, electrons also experience energy losses due to the synchrotron process and the inverse Compton scattering of radiation from the massive star and the surface of the neutron star. These energy losses determine the maximum energies of accelerated electrons, since their Larmor radius is typically far smaller than the characteristic dimensions of the considered scenario, i.e. $R_{\mathrm{L}}<R_{\mathrm{A}}$. This last condition in principle allows the acceleration of electrons up to $E_{\mathrm{e}} \approx 2 M_{16}^{4 / 7} B_{12}^{-8 / 7} \mathrm{PeV}$. However, as we show below, energy losses of electrons limit their maximum energies to significantly below this value.

Electrons lose energy with the IC process in the Thomson (T) and the Klein-Nishina (KN) regimes. In general, this process can affect the stellar radiation and the thermal X-ray radiation from the surface of the polar cap on the NS. We estimate the photon energy densities from the $\operatorname{star}\left(\rho_{\star}\right)$ and the polar cap $\left(\rho_{\text {cap }}\right)$ at the acceleration region to be,

$\rho_{\star}=\frac{4 \sigma T_{\star}^{4}}{c}\left(\frac{R_{\star}}{D}\right)^{2} \approx 6.1 \times 10^{3} T_{4}^{4}\left(\frac{R_{\star}}{D}\right)^{2} \mathrm{erg} \mathrm{cm}^{-3}$,

(where $T_{\star}=3 \times 10^{4} T_{4} \mathrm{~K}$ and $\sigma$ is the Stefan-Boltzmann constant) and,

$\rho_{\text {cap }}=\frac{4 \sigma T_{\text {cap }}^{4}}{c} \frac{R_{\text {cap }}^{2}}{R_{\mathrm{A}}^{2}} \approx 1.4 \times 10^{8} M_{16}^{11 / 7} B_{12}^{-8 / 7} \mathrm{erg} \mathrm{cm}^{-3}$,

where $T_{\text {cap }}=10^{7} T_{7} \mathrm{~K}$ is the temperature of the polar cap on the NS surface, and $R_{\text {cap }}$ is the polar cap radius on the NS surface to which the matter accretes. In Eq. (13), we assumed that the observed X-ray thermal emission is re-radiated from the region of the polar cap according to $L_{\mathrm{X}}=\pi R_{\text {cap }}^{2} \sigma T_{\text {cap. }}^{4}$. In fact, the emission from the polar cap region is well described by a black body spectrum (see e.g. Zane et al. 2000). The radius of the polar cap region on the NS surface, onto which the matter falls and from which the thermal X-ray emission is emitted, can be estimated from (assuming dipole structure of the magnetic field),

$R_{\text {cap }}=\left(R_{\mathrm{NS}}^{3} / R_{\mathrm{A}}\right)^{1 / 2} \approx 5 \times 10^{4} B_{12}^{-2 / 7} M_{16}^{1 / 7} \mathrm{~cm}$.

The surface temperature of the polar cap is then,

$T_{\text {cap }}=\left(L_{\mathrm{X}} / \pi R_{\text {cap }}^{2} \sigma\right)^{1 / 4} \approx 4.7 \times 10^{7} B_{12}^{1 / 7} M_{16}^{5 / 28} \mathrm{~K}$.

We note that, the energy density of radiation from the polar cap at the distance of the turbulent region $\left(R_{\mathrm{A}}\right)$ can be expressed explicitly by the basic parameters of the model.

We also estimate the energy density of the magnetic field at the transition region $\left(R_{\mathrm{A}}\right.$ given by Eq. (3)) to be,

$\rho_{\mathrm{B}}=B_{\mathrm{A}}^{2} / 8 \pi \approx 10^{7} M_{16}^{12 / 7} B_{12}^{-10 / 7} \mathrm{erg} \mathrm{cm}^{-3}$,

The energy losses of electrons due to each of the considered process (synchrotron and IC in the T regime) can be calculated from,

$\dot{P}_{\text {loss }}=(4 / 3) c \sigma_{\mathrm{T}} \rho \gamma^{2} \approx 2.7 \times 10^{-14} \rho_{(\mathrm{cap}, \mathrm{B}, \star)} \gamma^{2} \mathrm{erg} \mathrm{s}^{-1}$,

where $\sigma_{\mathrm{T}}$ is the Thomson cross section.

The energy losses of electrons emitted as radiation from the NS surface can dominate over energy losses from other targets only at low energies, i.e. when the scattering occurs in the $\mathrm{T}$ regime for Lorentz factors of electrons equal to, $\gamma_{\mathrm{T} / \mathrm{KN}}^{\mathrm{X}}<$ $m_{\mathrm{e}} c^{2} /\left(3 k T_{\star}\right) \approx 200 T_{7}^{-1}$. In the $\mathrm{KN}$ regime, the energy losses can be estimated approximately by introducing into Eq. (17) the value of the Lorentz factor corresponding to the transition between the $\mathrm{T}$ and $\mathrm{KN}$ regimes, $\gamma_{\mathrm{T} / \mathrm{KN}}^{\mathrm{X}}$. For the range of parameters defining the model, the IC energy losses of electrons in the $\mathrm{KN}$ regime becomes lower than the synchrotron energy losses for Lorentz factors about a factor of ten higher than $\gamma_{\mathrm{T} / \mathrm{KN}}^{\mathrm{X}}$ (typically 

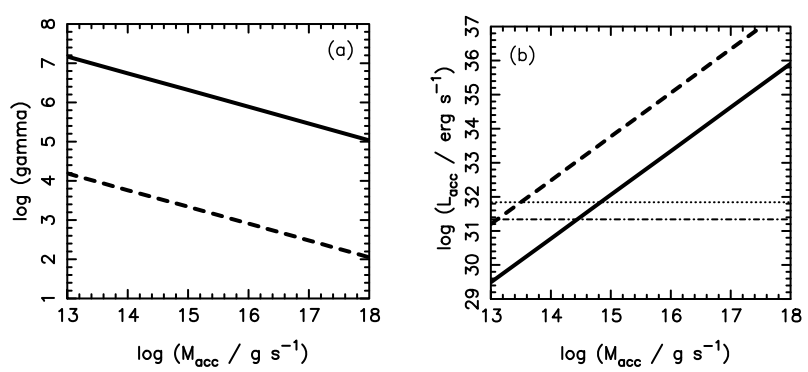

Fig. 2. The maximum energies of accelerated electrons a) and the maximum power that can be transferred to these electrons b) as a function of the accretion rate of the matter from the massive star onto the neutron star. The results are shown for the neutron stars that have the surface magnetic field $B=10^{12} \mathrm{G}$ (solid line), and $B=10^{9} \mathrm{G}$ (dashed). The acceleration parameter is equal to $\xi_{-1}=1$. The estimated Fermi LAT sensitivity at $1 \mathrm{GeV}(1 \mathrm{yr})$ for the source at the distance of $1 \mathrm{kpc}$ is $L_{\gamma} \approx 10^{32} \mathrm{erg} \mathrm{s}^{-1}$ (thin dot-dashed line) and CTA sensitivity (50 h) at $20 \mathrm{GeV} L_{\gamma} \approx 3 \times 10^{32} \mathrm{erg} \mathrm{s}^{-1}$ (thin dotted line).

at $\sim \mathrm{GeV}$ energies). Therefore the maximum energies of accelerated electrons are determined by the balance between energy gains from the acceleration process (Eq. (11)) and energy losses due to synchrotron processes (Eq. (17)),

$\gamma_{\max } \approx 3 \times 10^{5} \xi_{-1}^{1 / 2} B_{12}^{5 / 14} M_{16}^{-3 / 7}$.

It is clear that electrons can reach even TeV energies (see Fig. 2a) for realistic parameters of the model. However, $\mathrm{TeV} \gamma$-ray production will be strongly suppressed by synchrotron energy losses of electrons with $\mathrm{TeV}$ energies.

We can also estimate the characteristic energies of synchrotron photons expected in such a model by applying the approximate formula,

$\epsilon_{\mathrm{x}} \approx m_{\mathrm{e}} c^{2}\left(B_{\mathrm{A}} / B_{\mathrm{cr}}\right) \gamma_{\max }^{2} \approx 1.9 \xi_{-1} \mathrm{MeV}$,

where $m_{\mathrm{e}}$ is the electron rest mass, $B_{\mathrm{cr}}=4.4 \times 10^{13} \mathrm{G}$ is the critical magnetic field, and the Lorentz factor of electrons $\gamma_{\max }$ is given by Eq. (18). We conclude that synchrotron emission can extend throughout the soft $\gamma$-ray energy range and it should be detectable by satellites sensitive to these energies, e.g. the INTEGRAL observatory.

We note that the bremsstrahlung energy losses of relativistic electrons in the matter inside the transition region can be neglected since their energy-loss rate is relatively low,

$\dot{P}_{\mathrm{br}}=m_{\mathrm{e}} c^{3} \rho_{\mathrm{H}} \gamma / X_{\mathrm{o}} \approx 6.5 \times 10^{-5} \rho_{17} \gamma \mathrm{erg} \mathrm{s}^{-1}$,

where $X_{\mathrm{o}}=62 \mathrm{~g} \mathrm{~cm}^{-2}$ is the radiation length for hydrogen, and $\rho_{\mathrm{H}}=10^{17} \rho_{17} \mathrm{~cm}^{-3}$ is the density of matter. We can neglect the bremsstrahlung energy losses with respect to other energy loss rates, since the typical density of matter in the transition region is $\rho \approx 1.5 \times 10^{13} B_{12}^{-6 / 7} M_{16}^{10 / 7} \mathrm{~cm}^{-3}$ (estimated for the known accretion rate and the location of $\left.R_{\mathrm{A}}\right)$.

\subsection{Energetics}

The maximum power available for acceleration of electrons is limited by the energy extracted from the rotating neutron star by the infalling matter. This matter from the stellar wind has to be accelerated to the velocity of the magnetic field lines at $R_{\mathrm{A}}$ in order to accrete farther onto the neutron-star surface. The power that must be transferred from the rotating NS to the matter can be estimated from

$L_{\text {acc }}=\dot{M}_{\text {acc }} v_{\text {rot }}^{2} / 2 \approx 3 \times 10^{34} B_{12}^{8 / 7} M_{16}^{3 / 7} P_{1}^{-2} \mathrm{erg} \mathrm{s}^{-1}$.
By using the limiting period given by Eq. (8), we derive the upper limit to the available power in the accretor stage,

$L_{\text {acc }}<2.2 \times 10^{33} B_{12}^{-4 / 7} M_{16}^{9 / 7} \mathrm{erg} \mathrm{s}^{-1}$.

This power is shown in Fig. 2b for two example surface magnetic-field strengths of the neutron star (classical NS: $B=$ $3 \times 10^{12} \mathrm{G}$ and millisecond NS: $B=10^{9} \mathrm{G}$ ) as a function of the accretion rate. We assume that only fraction, $\eta$, of this power (typically $\eta=0.1$ ) can be converted to relativistic electrons in the turbulent and magnetized plasma at $R_{\mathrm{A}}$. We note that for reasonable accretion rates this power is higher than the minimum required power of the $\mathrm{GeV} \gamma$-ray source detectable by the Fermi LAT telescope and possibly also by the planned next generation Cherenkov telescopes system CTA, whose sensitivities should be about one order of magnitude higher than presently available.

The model discussed above for the accretor stage of the binary system is also applicable to the propeller stage in which only a fraction of the matter can eventually accrete onto the surface of the NS. However, in this case it is difficult to estimate the power transferable to relativistic particles due to the unknown observational signatures of matter accumulating close to the shock region.

In summary, electrons can be accelerated even to $\mathrm{TeV}$ energies in the case of neutron stars inside X-ray binaries with relatively weak thermal $\mathrm{X}$-ray emission from their surface, in contrast to the powerful X-ray binaries for which only a few tens $\mathrm{GeV}$ energy electrons are expected. However, the situation is the opposite concerning the maximum power transferred to these relativistic electrons. Sources in which electrons are accelerated only to $\mathrm{GeV}$ energies should be powerful enough to be observable by the $\gamma$-ray telescopes.

\section{Production of radiation}

We assume that electrons are accelerated in the turbulent transition region with a power-law spectrum to maximum energies estimated from Eq. (18). Eventually, the spectrum of electrons can be strongly peaked at the highest possible energies due to the synchrotron energy losses during acceleration process (the so called pile-up mechanism: see e.g. Protheroe 2004). As shown above, electrons lose energy by means of different radiation mechanisms of which the dominant ones are the synchrotron process (dominates at the highest energies) and the ICS of thermal radiation from the NS polar cap (which contributes at the high energies in the $\mathrm{KN}$ regime and dominates at lower energies in the T regime). We neglect the production of $\gamma$-rays by electrons in the acceleration region in the scattering of stellar radiation since its energy density can be safely neglected with respect to the energy density of the magnetic field (at high energies) and the energy density of polar cap radiation (at low energies) (see Sect. 2.1). To confirm whether electrons can lose energy efficiently already in the transition region, we estimate the convection timescale when matter is falling onto the NS surface along the open magnetic-field lines to be,

$\tau_{\text {conv }}=R_{\mathrm{A}} / v_{\text {rot }}=P_{1} / 2 \pi \mathrm{s}$.

We compare this timescale with the radiation timescale, which (for electrons with sufficiently low energies) is determined by the IC losses (in T regime) in the radiation field from the polar cap of the NS,

$\tau_{\mathrm{rad}}=m_{\mathrm{e}} \gamma / \dot{P}_{\mathrm{rad}} \approx 0.2 M_{16}^{-11 / 7} B_{12}^{8 / 7} \gamma^{-1} \mathrm{~s}$. 
From this comparison, we estimate the lower limit to the Lorentz factor of electrons losing energy efficiently before being convected onto the NS surface to be,

$\gamma=1.3 M_{16}^{-11 / 7} B_{12}^{8 / 7} P_{1}^{-1}$.

We conclude that relativistic electrons lose their energy close to the place of acceleration.

To calculate the $\gamma$-ray spectra produced by electrons inside the transition region, we simulate the energy-loss process of electrons in the radiation field of the polar cap and at the magnetic field of the transition region by applying the Monte Carlo method. It is assumed that the magnetic field is turbulent in the transition region (the distribution of electrons is isotropic) and that the radiation field originates in the region of the polar cap. For some range of model parameters, IC $\gamma$-rays can be absorbed farther into the radiation field of the polar cap. In this way the IC $\mathrm{e}^{ \pm}$pair cascade initiates. To determine the conditions for the cascade process, we calculate the optical depths for $\gamma$-ray photons in the radiation of the polar cap in Sect. 4. We also calculate the optical depths of $\gamma$-rays in the radiation field of the massive companion to check whether this $\gamma$-ray absorption process should also be taken into account when evaluating the $\gamma$-ray spectra escaping from the binary system.

\section{Optical depths for $\gamma$-rays}

As we argued above, the cooling of electrons in strong magnetic and radiation fields occurs efficiently. High energy $\gamma$-ray produced in the ICS of thermal photons from the polar cap can also be absorbed in this same radiation field and, in principle, also in the radiation field of the close massive companion star. Below, we calculate the optical depths of the $\gamma$-ray photons in these two radiation fields. We demonstrate that for specific conditions (determined mainly by the accretion rate onto the NS and the parameters of the NS), the $\gamma$-ray spectrum emerging from the binary system is in fact formed in the cascade process occurring in the radiation field of the polar cap.

\subsection{Polar cap radiation}

We calculate the optical depths for $\gamma$-rays by assuming that they are produced by electrons in the transition region at a distance $R_{\mathrm{A}}$ from the stellar surface. In these calculations, we assume the diluted black-body spectrum for the polar-cap emission with the dilution factor at the production site of $\gamma$-rays estimated to be $\left(R_{\text {cap }} / R_{\mathrm{A}}\right)^{2}$. The average optical depths (averaged isotropically for injected $\gamma$-rays) for different accretion rates onto the neutron star (i.e. equivalent to different $\mathrm{X}$-ray luminosities, see Eq. (1)) are shown in Fig. 3. It is clear that for low accretion rates $\left(<10^{16} \mathrm{~g} \mathrm{~s}^{-1}\right)$, when electrons can be accelerated to $\sim \mathrm{TeV}$ energies, the optical depths for $\gamma$-ray photons are relatively low, i.e. they are below unity for energies above $\sim 10 \mathrm{GeV}$. In this case, $\mathrm{GeV}-\mathrm{TeV} \gamma$-rays can escape from the radiation field of the polar cap without significant absorption.

On the other hand, for high accretion rates $\left(>10^{16} \mathrm{~g} \mathrm{~s}^{-1}\right)$, electrons are accelerated at most to a few tens of $\mathrm{GeV}$. The $\gamma$-rays produced by these electrons should be efficiently absorbed by means of the radiation of the polar cap. Therefore, in this case the escaping $\gamma$-ray spectra can be obtained only by calculating complicated IC $\mathrm{e}^{ \pm}$pair cascade within the radiation originating in the polar cap. We conclude that for high accretion rates, $\gamma$-rays, produced in this model, could be observable by the satellite telescopes (Fermi LAT and possibly AGILE).
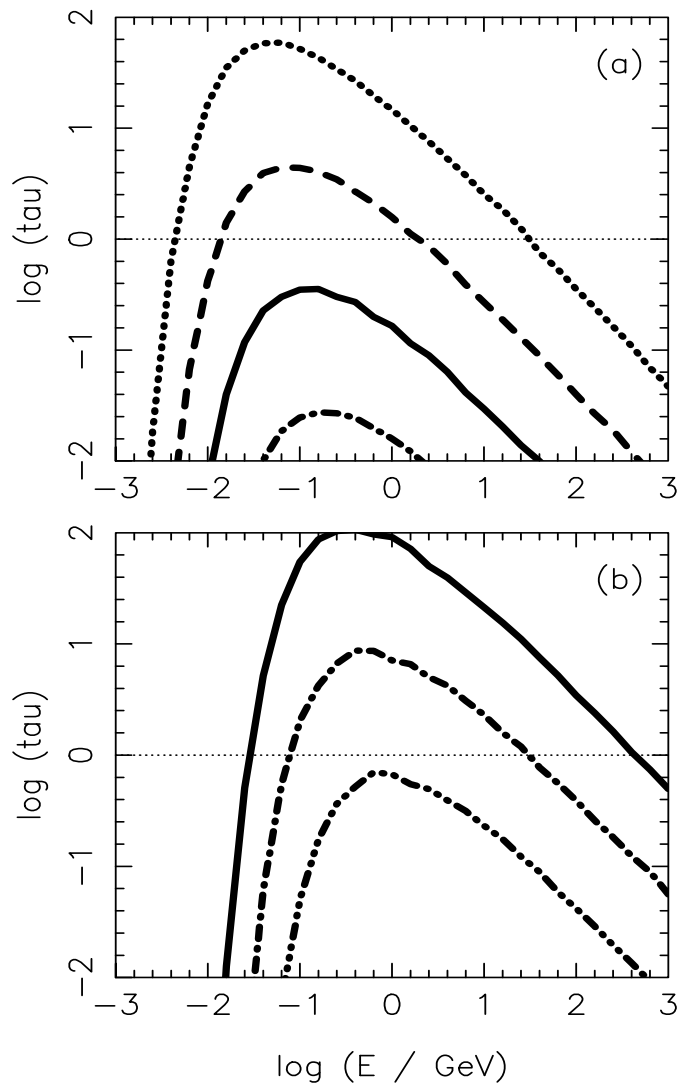

Fig. 3. The optical depths for $\gamma$-rays (averaged isotropically injected for all $\gamma$-rays) in the thermal radiation from the polar cap onto the NS surface as a function of $\gamma$-ray energies and for different accretion rates of matter onto the polar cap: $\dot{M}_{\text {acc }}=10^{18} \mathrm{~g} \mathrm{~s}^{-1}$ (dotted curve), $10^{17} \mathrm{~g} \mathrm{~s}^{-1}$ (dashed), $10^{16} \mathrm{~g} \mathrm{~s}^{-1}$ (solid), $10^{15} \mathrm{~g} \mathrm{~s}^{-1}$ (dot-dashed), and $10^{14} \mathrm{~g} \mathrm{~s}^{-1}$ (triple dot-dashed). The neutron star has the surface magnetic field equal to $B_{\mathrm{NS}}=3 \times 10^{12} \mathrm{G}$ (upper figure) and $10^{9} \mathrm{G}$ (bottom). The distance of the acceleration region from the polar cap $\left(R_{\mathrm{A}}\right)$ is defined by the above parameters. The thin dotted line marks the optical depth equal to unity.

\subsection{Massive-star radiation}

The optical depths at $\gamma$-ray photons injected at an arbitrary distance from the surface of the massive star were calculated for the first time in the general case (including also dimensions of the star) by Bednarek $(1997,2000)$. The optical depths for $\gamma$-rays close to the surface of other stars can be easily rescaled from those early calculations. For example, $\gamma$-ray photons with energies, $E_{\gamma}^{\mathrm{o}}$, propagating at specific distances $D$ and in directions (defined by the angle $\alpha$ ) close to the star with specific parameters $\left(T_{\mathrm{o}}\right.$ and $\left.R_{\mathrm{o}}\right)$ are related to the optical depths around arbitrary stars with $T_{\star}$ and $R_{\star}$ in the following way,

$\tau\left(E_{\gamma}^{\star}=\frac{E_{\gamma}^{\mathrm{o}}}{S_{\mathrm{T}}}, T_{\star}, R_{\star}, D, \alpha\right)=S_{\mathrm{T}}^{3} S_{\mathrm{R}} \tau\left(E_{\gamma}^{\mathrm{o}}, T_{\mathrm{o}}, R_{\mathrm{o}}, D, \alpha\right)$

where $S_{\mathrm{T}}=T_{\star} / T_{\mathrm{O}}, S_{\mathrm{R}}=R_{\star} / R_{\mathrm{O}}$, and the distance $D$ from the stellar surface of a specific star is measured in stellar radii. The example calculations of the optical depths for the case of one specific massive star (present inside the binary system IGR J19140+098) are shown in Fig. 4. These optical depths become higher than unity for $\gamma$-rays with energies above $\sim 20 \mathrm{GeV}$. As we show later, $\gamma$-rays produced in the considered model have energies typically below $\sim 10 \mathrm{GeV}$. Their absorption in the radiation field of the massive star can be safely neglected. Therefore, the absorption effects of $\gamma$-rays should not introduce any 

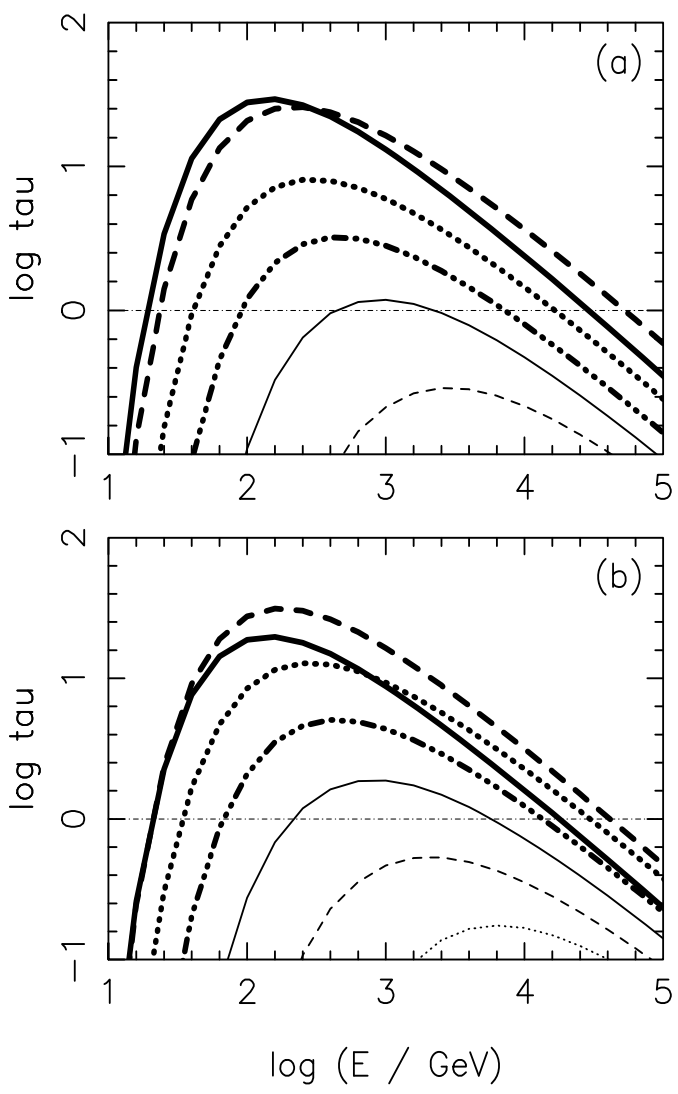

Fig. 4. The optical depths as a function of $\gamma$-ray energies are shown for the case of the massive star in the massive binary IGR J19140+098 discovered by INTEGRAL telescope: $R_{\star}=30 R_{\odot}, T_{\star}=2.8 \times 10^{4} \mathrm{~K}$ and two distances from the star $D=3 R_{\star}$ a) and $2 R_{\star}$ b). Specific curves show the optical depths for the injection angles $\alpha=0^{\circ}$ (thick solid curve), $30^{\circ}$ (thick dashed), $60^{\circ}$ (thick dotted), $90^{\circ}$ (thick dot-dotdashed), $120^{\circ}$ (thin solid), $150^{\circ}$ (thin dashed), and $180^{\circ}$ (thin dotted), measured from the direction towards the star.

modulation of the $\gamma$-ray signal with the period of the binary in the case of accreting neutron stars. This is in contrast to the $\mathrm{TeV} \gamma$-ray binaries that are supposed to contain ejecting neutron stars (a strong pulsar wind prevents the accretion of stellar wind: see e.g. the model considered by Sierpowska \& Bednarek 2005). The eventual modulation of the $\gamma$-ray signal with the period of the binary can be only produced by the change of the accretion rate with the distance of the neutron star on its elliptic orbit around the massive star.

\section{Gamma-rays from the vicinity of an accreting neutron star}

In the considered model, radiation is produced by electrons accelerated to their maximum energies, estimated by Eq. (18) for the synchrotron and IC processes. Synchrotron energy losses dominate at the highest electron energies (close to $E_{\max }$ ), and the IC process can dominate only at lower energies due to the KN cross section. However, as we have shown above, for high accretion rates, $\gamma$-rays produced by electrons can be absorbed farther by the thermal radiation from the NS surface. In Fig. 3 we show the average optical depths for the $\gamma$-rays in the thermal radiation from the NS polar cap in order to envisage for what parameters the cascading effects can become important. Therefore, in these cases we must consider the production of the $\gamma$-rays in the IC $\mathrm{e}^{ \pm}$pair cascade with additional synchrotron energy losses of primary electrons and secondary $\mathrm{e}^{ \pm}$pairs. On the other hand, for low accretion rates, the cascade does not develop, and $\gamma$-rays are produced only as a result of the cooling of primary electrons. To follow the process of $\gamma$-ray production, we developed a numerical code that simulates the cooling process of electrons by taking into account not only the $\gamma$-ray production in the IC process but also the synchrotron-energy losses of primary electrons and secondary cascade $\mathrm{e}^{ \pm}$pairs. We note that the acceleration of electrons occurs inside the inner pulsar magnetosphere, where the magnetic field is relatively strong and depends on the distance of the turbulent transition region from the NS surface. Since the synchrotron process dominates over the IC process in the high energy part of the injected electron spectrum, the $\gamma$-rays are rarely produced with energies comparable to the energies of primary electrons. Our IC $\mathrm{e}^{ \pm}$pair cascade code with synchrotron-energy losses provides both the synchrotron and the IC spectra expected in such a model.

Two models for injection of relativistic electrons are considered:

1. electrons injected with a power-law spectrum up to a maximum Lorentz factor $\gamma_{\max }$ (see Eq. (18)), as expected in the stochastic acceleration mechanism. The power-law spectrum of primary electrons is normalized to correspond to a fraction, $\eta$, of the kinetic power transferred from the rotating neutron star to the matter in the transition region;

2. electrons injected with the Lorentz factors $\gamma_{\max }$. This injection spectrum provides a good approximation to the cases of acceleration processes occurring with large radiativeenergy losses (the accumulation mechanism at the high end of accelerated power law spectrum of electrons; see e.g. Protheroe 2004).

These two injection models are likely to represent the limiting cases of the true acceleration process of electrons in the turbulent region of the matter accreting onto the magnetized neutron star.

We investigate two general scenarios describing the cases of pure accretor phase (where all the matter arriving at the transition region falls onto the NS surface) and the intermediate accretor-propeller phase (where only part of the matter arriving at the transition region accretes onto the NS surface and the remainder is expelled from the vicinity of the NS by the propeller mechanism).

\subsection{The accretor stage}

The scenario for the accretion process onto a neutron star in the accretor stage is far more precisely defined, since the thermal radiation field from the NS surface (depending on the dimensions of the hot spot and its temperature) is uniquely determined by the parameters describing the model. Therefore, the synchrotron and IC spectra produced by electrons depends on a relatively small number of free parameters, i.e. the accretion rate onto NS, the surface magnetic field of NS, and the acceleration rate $\xi$. The observed X-ray to $\gamma$-ray power depends additionally on the parameter, $\eta D^{-2}$, which combines the energy-conversion efficiency from the transition region to relativistic electrons, $\eta$, and the distance to the source $D=1 D_{1} \mathrm{kpc}$. At first, we performed calculations for the power-law spectrum of injected electrons $N(\mathrm{e})=A E^{-s}$ between $E_{\max }$ and $E_{\min }$, where $A$ is the normalization coefficient that equals $A=\eta L_{\mathrm{acc}}(2-s) /\left(E_{\max }^{(2-s)}-E_{\min }^{(2-s)}\right)$. We note, that only $A$ depends weakly on $E_{\min }$ for the spectral indices of approximately 2 . In our example calculations, we apply a spectral index equal to 2.2 and select $E_{\text {min }}=30 \mathrm{MeV}$, since 

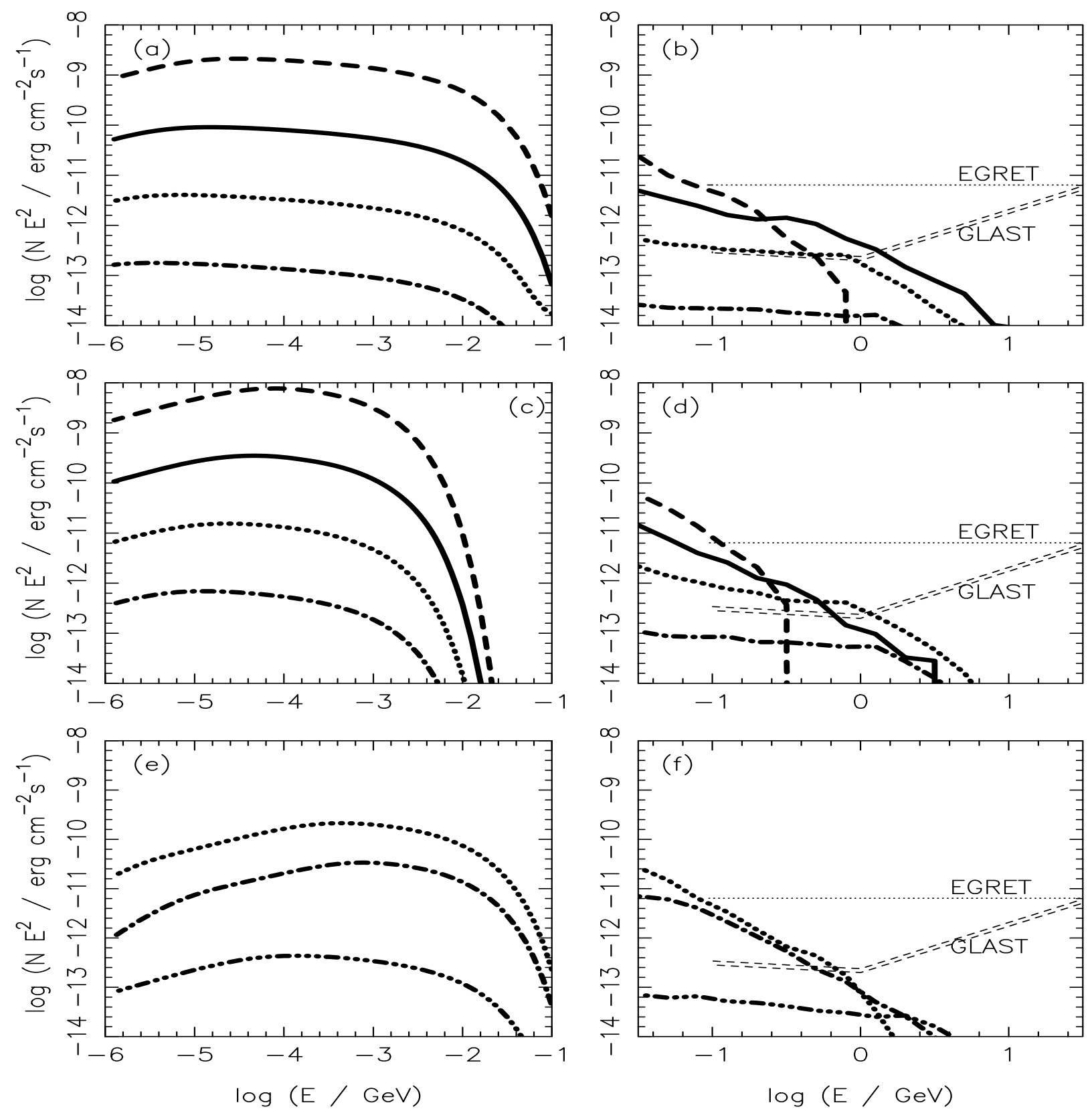

Fig. 5. The IC $\gamma$-ray spectra (on the right) and synchrotron spectra (on the left) ( $N E^{2}-$ the differential spectra multiplied by energy squared) produced in a sequence of radiation processes by relativistic electrons. The IC process occurs in the thermal radiation field produced by the matter accreting onto the neutron-star surface. The X-ray and $\gamma$-ray spectra are shown for different accretion rates: $M=10^{18} \mathrm{~g} \mathrm{~s}^{-1}$ (dashed curve), $10^{17} \mathrm{~g} \mathrm{~s}^{-1}$ (solid), $10^{16} \mathrm{~g} \mathrm{~s}^{-1}$ (dotted), $M_{\text {acc }}=10^{15} \mathrm{~g} \mathrm{~s}^{-1}$ (dot-dashed), $10^{14} \mathrm{~g} \mathrm{~s}^{-1}$ (triple dot-dashed). The spectra are shown for the acceleration parameter $\xi=0.1$, the surface magnetic field of the NS $B_{\mathrm{NS}}=10^{12} \mathrm{G}$ (upper figures), $\xi=0.01$ and $B_{\mathrm{NS}}=10^{11} \mathrm{G}$ (middle), and $\xi=0.1$ and $B_{\mathrm{NS}}=10^{9} \mathrm{G}$ (bottom). The spectrum of accelerated electrons is of the power-law type $\left(\mathrm{d} N(E) / \mathrm{d} E \propto E^{-s}\right)$ with the spectral index $s=2.2$ between $30 \mathrm{MeV}$ and the maximum energy given by Eq. (18). The factor determining the power transferred from the transition region to relativistic electrons depends on the energy-conversion efficiency, $\eta$, and the distance to the source, $D=1 D_{1} \mathrm{kpc}$. It is assumed to be equal to $\eta D_{1}^{-2}=0.1$. The period of the pulsar is equal to the limiting value given by Eq. (8). The level of sensitivity of the the EGRET and GLAST telescopes are marked by thin dotted and double-dashed lines.

we reduce the energies of these electrons only to these minimum energies. Electrons with such energies are unable to produce $\gamma$-rays detectable by the Fermi LAT. The simulated spectra are also shown for different values of $\dot{M}, \xi$, and $B$ (see Fig. 5).

In Figs. $5 \mathrm{a}-\mathrm{d}$, we consider the case of a classical relatively young NS $\left(B \gg 10^{9} \mathrm{G}\right)$, rotating with the limiting period defined by Eq. (8). When considering the sensitivity of the Fermi LAT telescope, it becomes clear that $\gamma$-ray emission from these objects can be detected at energies below $\sim 1 \mathrm{GeV}$ only in the case of high accretion rates (significantly above $10^{16} \mathrm{~g} \mathrm{~s}^{-1}$ ). The power-law synchrotron spectra corresponding to these accretion rates should also be easily detected by soft and hard X-ray detectors presently on the orbit. Note that due to strong magnetic field and large energies of primary electrons, the synchrotron spectra can extend in some cases also through the soft $\gamma$-ray energy range. In Figs. 5e,f, we show the synchrotron and IC spectra for the case of a NS with a low surface magnetic field, characteristic of so-called millisecond pulsars. In the case of these slowly 

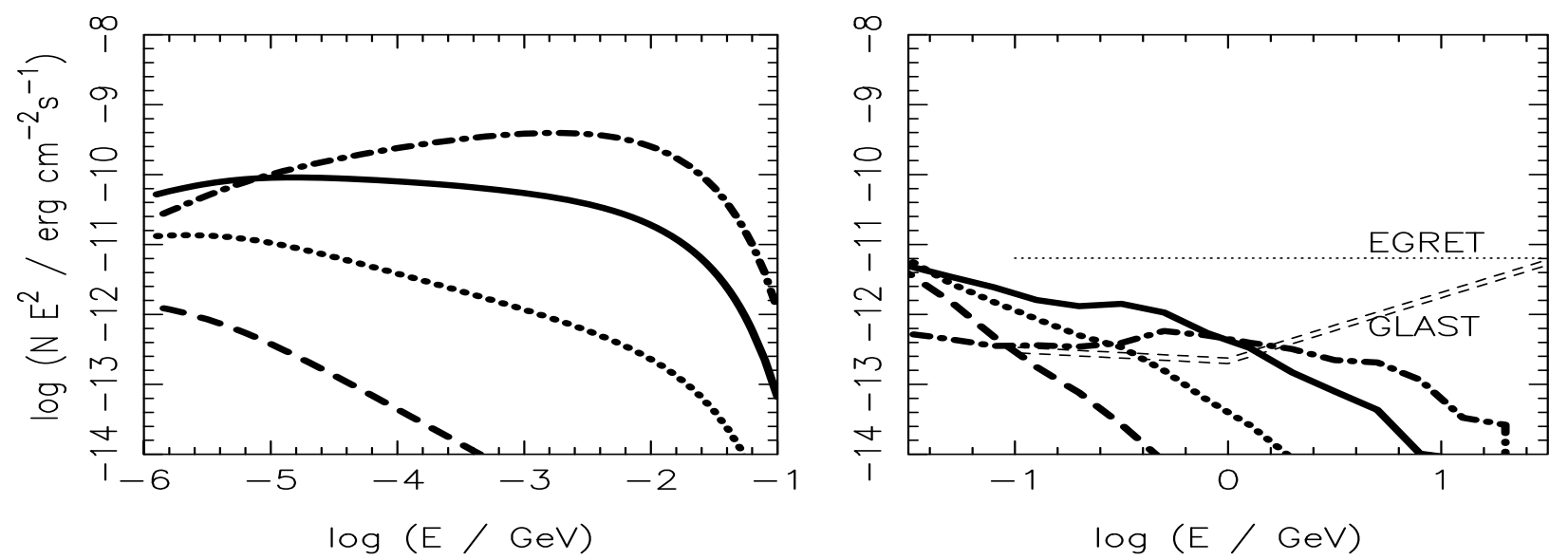

Fig. 6. As in Fig. 5 but for different spectral indexes of injected electrons: $s=4$ (dashed), 3 (dotted), 2.2 (solid), 1 (dot-dashed). The parameters describing the model are the following: $\xi=0.1$, the surface magnetic field of the NS $B_{\mathrm{NS}}=10^{12} \mathrm{G}$, and the accretion rate $M_{\text {acc }}=10^{17} \mathrm{~g} \mathrm{~s}$.

magnetized neutron stars, $\gamma$-ray emission can also be detected by the Fermi LAT telescope, provided that the accretion rate onto the NS is above a few $10^{14} \mathrm{~g} \mathrm{~s}^{-1}$. We conclude that $\gamma$-ray telescopes presently in orbit should be able to detect $\gamma$-ray emission of energies of a few hundred $\mathrm{MeV}$ from the class of accreting NS. These NS are characterized by thermal emission from its surface at a level of above $\sim 2 \times 10^{36} \mathrm{erg} \mathrm{s}^{-1}$ in the case of strongly magnetized neutron stars, and above a few $10^{34} \mathrm{erg} \mathrm{s}^{-1}$ in the case of a NS with a magnetic field characteristic of millisecond pulsars provided that the source is at a distance of $1 \mathrm{kpc}$. These limiting X-ray luminosities were derived by using the relation between the accretion rate and the thermal emission from the NS surface (see Eq. (1)).

We also investigate how the detectability of these accreting NSs depends on the spectral index of the injected electrons. In Fig. 6 the synchrotron and IC $\gamma$-ray spectra are shown for the range of spectral indexes where $s=1-4$ and other parameters describing the model have been fixed: $\dot{M}=10^{17} \mathrm{~g} \mathrm{~s}^{-1}, \xi=0.1$, and $B=10^{12} \mathrm{G}$. The electron spectrum must have a spectral index lower than $\sim 3$ to produce IC $\gamma$-rays above the sensitivity of Fermi LAT telescope. These spectral indexes are expected in stochastic models of particle acceleration in turbulent media.

As already noted above, the acceleration of particles experiencing significant radiative energy losses may result in their accumulation at the highest energies depending on the balance between energy gains from the acceleration mechanism and energy losses (see e.g. Protheroe 2004). In such cases, most of the energy is accumulated in particles with the highest possible energies. A first approximation, we can consider that the spectrum of particles is mono-energetic. Therefore, in Fig. 7 we also show the photon spectra expected in the model for the case of mono-energetic injection of electrons. In such a case, IC $\gamma$-ray spectra are flat. Their detectability is more difficult than in the case of electrons injected with a power-law spectrum. This is due to the fact that electrons with maximum possible energies lose most of their energy with synchrotron processes. Therefore, the IC spectra are reduced in intensity and synchrotron spectra are enhanced (compare the corresponding cases shown in Figs. 5 and 7). We conclude that an accreting NS, which has the highest probability of being detected by the Fermi LAT telescope, should have intermediate values of $\xi$ and $B$ and high accretion rates (see Figs. 7c,d). These parameters enable the acceleration of electrons to energies of the order of a few tens of $\mathrm{GeV}$, i.e. close to energies of which the ICS process begins to become of comparable efficiency to the synchrotron process.
The $\gamma$-ray photons produced by electrons in the transition region of the accretion flow onto the NS, originate close to the massive companion star creating the strong radiation field. In Sect. 4.2, we show the optical depths for $\gamma$-rays in the radiation field of the massive star with parameters typical of massive binary systems (the example case of the massive binary IGR J19140+0951). As we have shown above, primary electrons accelerated to the maximum energies reduce their energies by means of the synchrotron process. Therefore, $\gamma$-rays produced by them in the IC process have energies typically below $\sim 10 \mathrm{GeV}$. On the other hand, the optical depths of the massive star radiation (see Fig. 4) are quite high but at energies that are clearly above the energies of $\gamma$-rays produced in our model. Therefore, we conclude that for model considered here, the absorption of $\gamma$-rays in the stellar radiation can be neglected.

In Figs. 7a,c,e, we also display the spectra of thermal radiation from the NS polar-cap region for the example accretion rate of $M_{\mathrm{acc}}=10^{16} \mathrm{~g} \mathrm{~s}^{-1}$ and different values of the magnetic field on the NS surface (i.e. $B=10^{12} \mathrm{G}$ (a), $10^{11} \mathrm{G}(\mathrm{c})$, and $10^{9} \mathrm{G}(\mathrm{e})$. These spectra clearly dominate over the nonthermal synchrotron spectra produced by relativistic electrons below a few $\mathrm{keV}$. As we have already noted above, these soft X-ray thermal excesses have been reported from some INTEGRAL hard X-ray massive binaries. In reality, this soft thermal X-ray emission may be modified significantly due to the interaction with the matter accreting onto the NS surface, the matter of the stellar wind, and the matter on the surface of the close massive companion. In our calculations, we are unable to take these possible modifications into account.

\subsection{The intermediate accretor-propeller stage}

As noted above, in the pure propeller stage the matter arriving in the transition region at the Alfven radius, $R_{\mathrm{A}}$, is expelled by the centrifugal source from the vicinity of the neutron star preferentially along the rotational axis. Here we consider the intermediate case in which a small fraction of the accreting matter is able to penetrate the NS surface but majority is expelled outside the neutron star. It is unclear whether this process remains stationary in time or is only non-stationary when the accretion rate onto the neutron star varies with time e.g. due to the clumpy wind or the elliptic orbit of the NS around the massive star. To take account of the effects of only the partial accretion of matter onto the NS surface, we introduce the parameter, $\kappa$, which is the ratio 

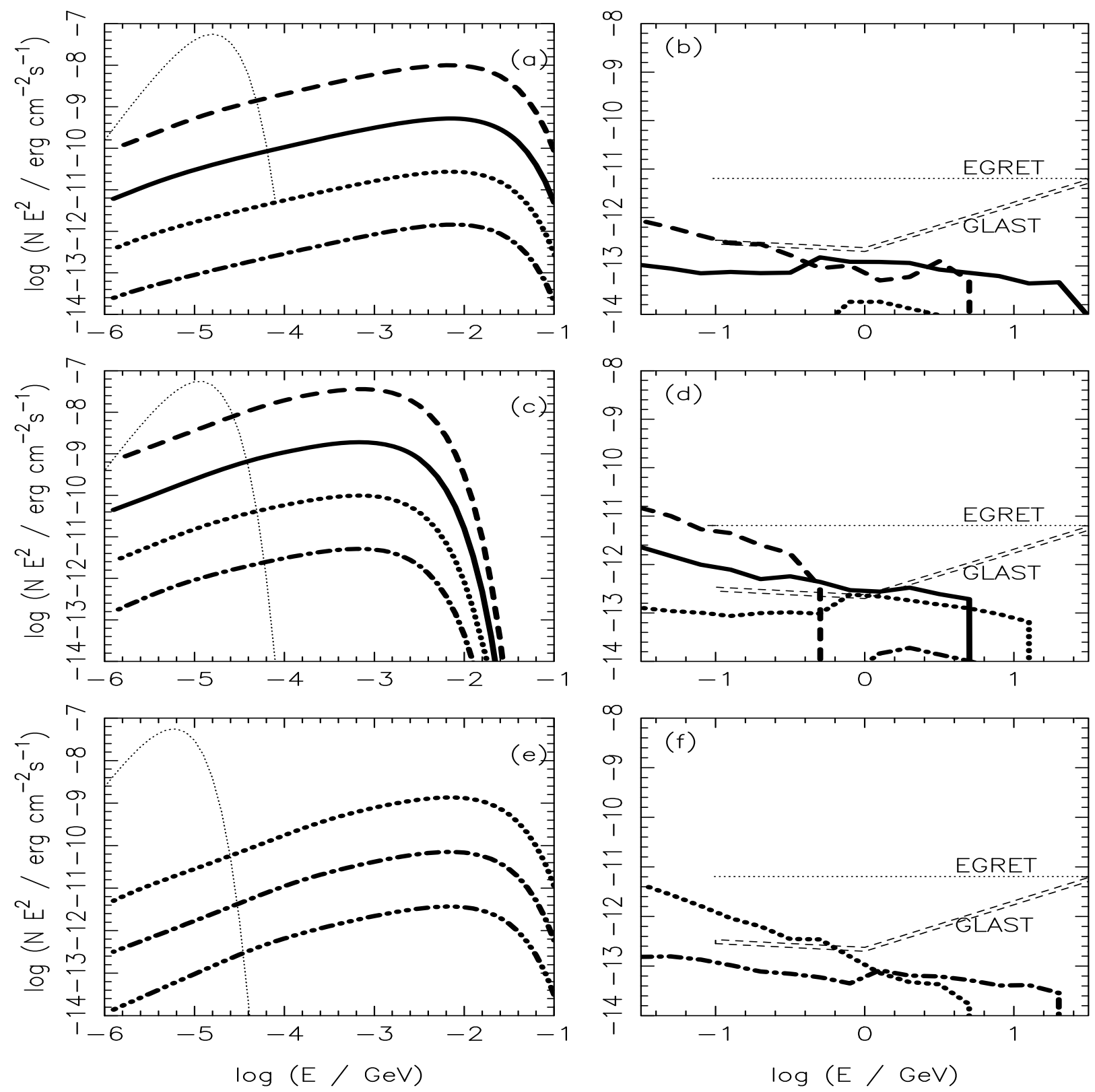

Fig. 7. As in Fig. 5 but for mono-energetic injection of electrons with energies given by Eq. (18). The specific curves show the results obtained for these same parameters as in Fig. 5. The spectrum of thermal radiation emitted from the neutron star surface is also shown for the typical accretion rate $M_{\text {acc }}=10^{16} \mathrm{~g} \mathrm{~s}^{-1}$ and corresponding surface magnetic-field strengths (thin dotted curves in a), $\mathbf{c}$ ), and e).

of the matter accreting onto the NS surface to the entire amount of matter arriving in the transition region (i.e. the matter accreted onto the surface and expelled from the vicinity of NS). In such an intermediate case, the temperature of the polar cap region on the NS surface (given by Eq. (23)) should be a factor of $\kappa^{1 / 4}$ lower than estimated in the case of complete accretion. However, in principle the power available for acceleration of electrons can be higher than expected from the period limiting of the accretor and propeller stage (given by Eq. (8)), and is in fact limited by Eq. (5). In conclusion, as a result of only the partial accretion of matter from the transition region onto the NS surface, the surface temperature of the polar cap is lower, and the power transferred to electrons can be higher than expected for the pure accretor stage.

In Fig. 8, we show the example $\gamma$-ray spectra obtained in the case of the partial accretion of matter onto the NS surface, assuming different accretion rates defined by the factor $\kappa$. We also scale the power transferred to the relativistic electrons by the factor $\kappa^{-1}$, since in this case more energy can be extracted from the transition region. Based on these calculations, we expect that the $\gamma$-ray spectra produced at the intermediate stage are steeper power-laws, although of higher luminosities. Therefore, they should be more easily detected by $\gamma$-ray telescopes (see Fig. 8 for a comparison at the sensitivity of the Fermi LAT telescope for a source at the distance of $1 \mathrm{kpc}$ ).

\section{Discussion and conclusions}

We have proposed that accreting neutron stars inside compact massive binary systems produce $\gamma$-ray fluxes that are detectable by the satellite telescope Fermi LAT. To demonstrate this, we have considered electron acceleration in the turbulent, stronglymagnetized, transition region in the inner neutron-star magnetosphere that appears as a result of the interaction between 

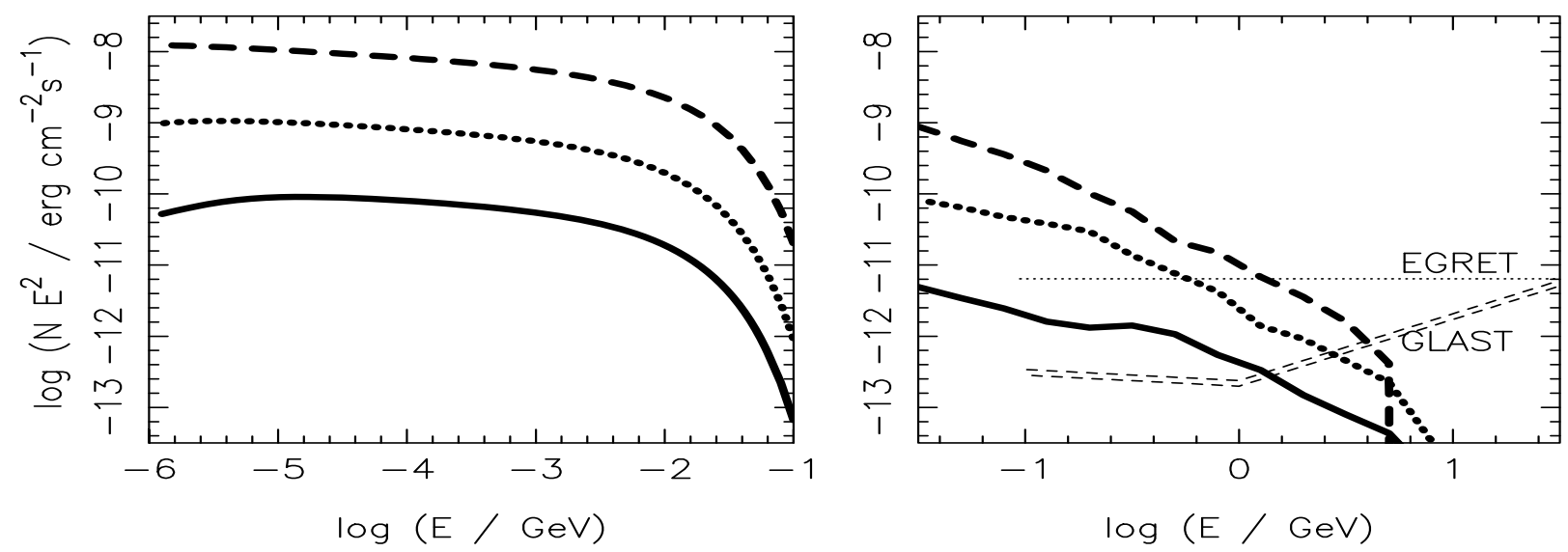

Fig. 8. As in Fig. 5 but for different rates describing the amount of the matter accreting onto the NS surface with respect to the entire amount of the matter arriving in the transition region (accreting and expelled from the vicinity of the NS in propeller mechanism): $\kappa=1$ (solid curve), 0.1 (dashed), and 0.01 (dotted). The basic parameters of the model are the following: $\dot{M}=10^{17} \mathrm{~g} \mathrm{~s}^{-1}, B=10^{12} \mathrm{G}$, and $\xi=0.1$.
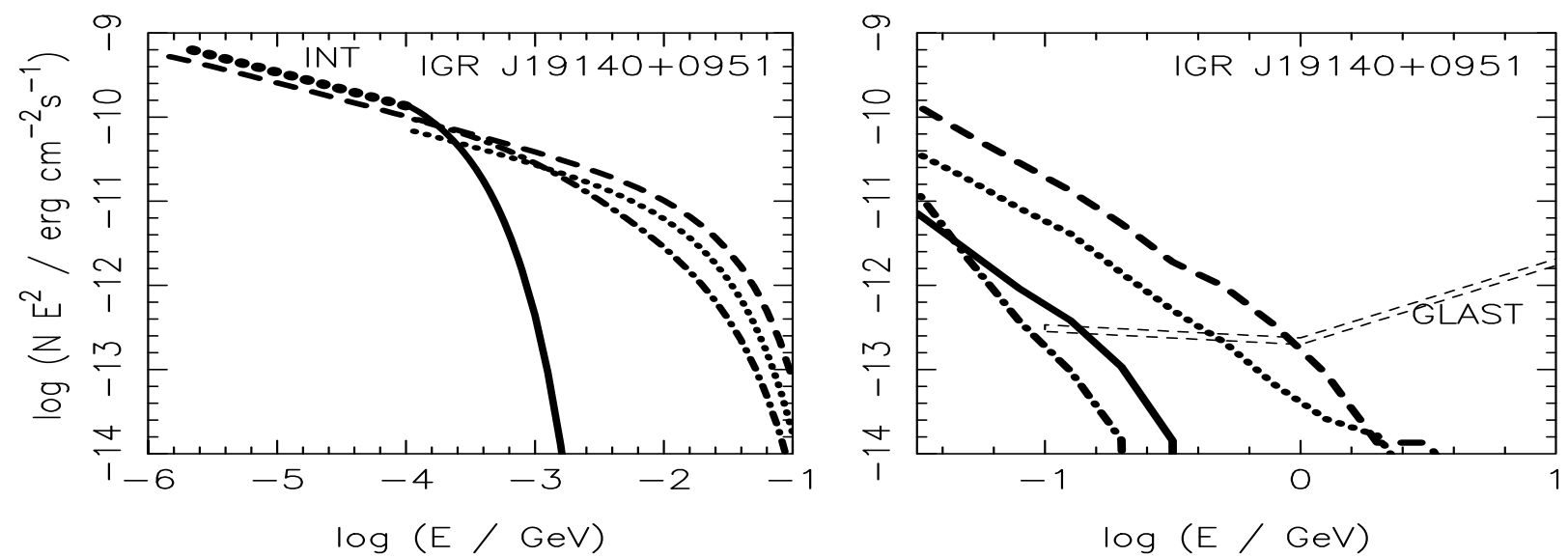

Fig. 9. a) The comparison of the hard X-ray spectra detected by the INTEGRAL observatory from the massive binary IGR J19140+0951 with the synchrotron spectra expected from the vicinity of a neutron star for the pure accretor model with the parameters: $\dot{M}=2 \times 10^{17} \mathrm{~g} \mathrm{~s}^{-1}, B=10^{11} \mathrm{G}$, and $\xi=0.1$, spectral index of electrons $s=2.8$ (solid curve), $\dot{M}=3 \times 10^{17} \mathrm{~g} \mathrm{~s}^{-1}, B=10^{9} \mathrm{G}$, and $\xi=0.1, s=4$ (dot-dashed), the intermediate, accretor-propeller models for: $\dot{M}=10^{17} \mathrm{~g} \mathrm{~s}^{-1}, B=10^{11} \mathrm{G}, \xi=0.1$, and $\kappa=0.1$ and $s=2.8$ (dotted), and $\dot{M}=10^{17} \mathrm{~g} \mathrm{~s}^{-1}, B=10^{12} \mathrm{G}, \xi=0.1$, and $\kappa=0.01$ and $s=2.8$ (dashed). b) The IC $\gamma$-ray spectra expected in the model for the afore mentioned parameters based on the normalization of the synchrotron spectra to the INTEGRAL observations in the X-ray energy range. The level of sensitivity of the Fermi LAT telescope is indicated by a double-dashed line.

accreting matter and a rotating NS magnetosphere. Relativistic electrons produce $\mathrm{X}$-rays and $\gamma$-rays as a result of synchrotron and IC processes corresponding to thermal radiation originating in the NS surface. The cooling process of electrons is followed by applying the Monte Carlo method, since some of the $\gamma$-rays produced can be absorbed farther by the thermal radiation. We have shown that synchrotron emission from primary electrons and secondary cascade $\mathrm{e}^{ \pm}$pairs can extend in energy up to the $\mathrm{MeV}$ energy range and $\mathrm{IC}$ emission can extend up to a few $\mathrm{GeV}$.

A physical realization of this scenario may correspond to a class of obscured, massive, binary systems discovered by the INTEGRAL observatory, which exhibit: (1) hard, power-law X-ray emission and; (2) evidence of a soft X-ray black-body component. As an example, we considered here in more detail the high mass X-ray binary system, IGR J19140+0951, discovered by INTEGRAL (Hannikainen et al. 2003). A compact object in this system is likely to be a neutron star (Cabanac et al. 2005). IGR J19140+0951 is at a distance of $\sim 2-3 \mathrm{kpc}$ (Rahoui et al. 2008). A hard X-ray emission up to $100 \mathrm{keV}$ with a spectral index $2.39 \pm 0.11$ is observed from this source (Hannikainen et al. 2004). Its X-ray luminosity in the high state was $\sim 3.7 \times 10^{37} \times(D / 10 \mathrm{kpc})^{2} \mathrm{erg} \mathrm{s}^{-1}$ (Rodriquez et al. 2005). Unfortunately, the basic parameters of the neutron star in IGR J19140+0951 are unknown. Therefore, we investigated the range of model parameters that are consistent with the hard power-law spectrum detected in the X-ray energy range by INTEGRAL. Comparison of the observations with the example calculations were shown in Fig. 9. It is clear that for some parameters the $\gamma$-ray flux predicted at a few hundred $\mathrm{MeV}$ is detectable by extensive Fermi LAT observations.

We performed calculations for neutron stars with parameters characteristic of the classical radio pulsars (surface magneticfield strength $B \sim 10^{12} \mathrm{G}$ and periods of the order of seconds) and for the millisecond pulsars $\left(B \sim 10^{9} \mathrm{G}\right.$, periods of a few to several milliseconds). It is clear that to produce $\gamma$-ray fluxes observable by the Fermi LAT telescope, the neutron stars in binary systems at a distance of a few kpc should collect the matter from the wind with a relatively high accretion rates $\left(>10^{16} \mathrm{~g} \mathrm{~s}^{-1}\right)$. However, in the case of millisecond pulsars these accretion rates can be significantly lower (just above a few $10^{14} \mathrm{~g} \mathrm{~s}^{-1}$ ). Therefore, in principle, the millisecond pulsars appear to be the most probable $\gamma$-ray sources. On the other hand, 
millisecond pulsars have only low mass companions, so high accretion rates are not expected for these binary systems.

We note that both synchrotron X-ray and IC $\gamma$-ray emission are produced in the models almost isotropically. As we have shown above, we do not expect any modulation of the $\gamma$-ray signal with the period of the binary system due to the selective absorption of the companion-star soft radiation, as expected in the case of TeV $\gamma$-ray binary systems (e.g. LS 5039 and LSI 61 303). However, the modulation might be related to the change in the accretion rate in the case of the elliptic orbit of the neutron star. It is expected that the accretion rate should increase when the neutron star is closer to the companion star. Therefore, we predict that the highest fluxes of X-rays and $\gamma$-rays should be observed closer to the periastron passage of the neutron star. Moreover, the X-ray emission should be correlated with the $\gamma$-ray emission (see spectra in Figs. 5-8).

In the model considered here, we do not take into account the scattering of non-thermal synchrotron radiation by the accelerated electrons. As we have shown in Fig. 7, the thermal radiation clearly dominates at energies below a few $\mathrm{keV}$ over the non-thermal synchrotron radiation produced by accelerated electrons and secondary cascade $\mathrm{e}^{ \pm}$pairs. Since their energy densities at the acceleration site scales in a similar way with distance from the NS, the energy losses by thermal radiation must dominate over the energy losses due to non-thermal radiation. Therefore, we can safely neglect the cooling of electrons by this non-thermal radiation. We also note, that the scattering of nonthermal X-rays with energies above a few $\mathrm{keV}$ is inefficient since it occurs in the Klein-Nishina regime.

Processes similar to these considered in this paper are also expected in the case of non-spherical accretion of matter onto a rotating neutron star. For example, in the case of disk accretion, the corresponding accretion rate should be scaled by a part of sphere that is intercepted by the accretion disk. Then, the turbulent region in the inner disk, in which acceleration of electrons occurs, is also limited to the corresponding part of the sphere. Only IC process may become more complicated in this case since thermal radiation from the hot spot on the neutron star surface, indicative of relativistic electrons, can be partially obscured by the neutron-star surface.

In conclusion, we predict that present satellite telescopes can discover a new class of $\gamma$-ray sources, i.e. accreting neutron stars inside binary systems. The emission from these sources should be characterized by a strong hard, synchrotron spectra and IC $\gamma$-rays limited to energies below $\sim 1 \mathrm{GeV}$, due to the significant synchrotron energy losses of relativistic electrons accelerated in the vicinity of neutron stars.

Acknowledgements. This work is supported by the Polish MNiSzW grant N N203 390834.

\section{References}

Baan, W. A., \& Treves, A. 1973, A\&A, 22, 421

Bednarek, W. 1997, A\&A, 322, 523

Bednarek, W. 2000, A\&A, 363, 646

Bednarek, W., \& Giovannelli, F. 2007, A\&A, 464, 437

Begelman, M. C., \& Rees, M. J. 1984, MNRAS, 206, 209

Chaty, S. 2008, Chin. J. Astron. Astrophys., 8, 197

Cabanac, C., Rodriquez, J., Petrucci, P.-O., et al. 2005, Chin. J. Astron. Astrophys., 5, 93

Corbet, R. H. D., Hannikainen, D. C., \& Remillard, R. 2005, ATel, 269

Hannikainen, D. C., Rodriquez, J., \& Pottschmidt, K. 2003, IAUC, 8088

Hannikainen, D. C., Rodriquez, J., Cabanac, C., et al. 2004, A\&A, 423, L17

Hannikainen, D. C., Rawlings, M. G., Muhli, P., et al. 2007, MNRAS, 380, 665

Lipunov, V. M. 1992, Astrophysics of neutron stars (Heidelberg: SpringerVerlag)

Protheroe, R. J. 2004, APh, 21, 415

Rodriquez, J., \& Bodaghee, A. 2008, in The Nature and Evolution of X-ray Binaries in Diverse Environments, ed. S. Bandyopadhyay, et al. [arXiv:0801.0961]

Rodriquez, J., Cabanac, C., Hannikainen, D. C., et al. 2005, A\&A, 432, 235

Rahoui, F., Chaty, S., Lagage, P.-O., \& Pantin, E. 2008, A\&A, 484, 801

Sierpowska, A., \& Bednarek, W. 2005, MNRAS, 356, 711

Tavani, M. 1991, ApJ, 379, L69

Tavani, M. 1993, A\&AS, 97, 313

Tavani, M., \& Brookshaw, L. 1993, ApJ, 413, L39

Treves, A., Colpi, M., \& Lipunov, V. M. 1993, A\&A, 269, 319

Zane, S., Turolla, R., \& Treves, A. 2000, ApJ, 537, 387 